\title{
Veränderung von Interesse und Selbstwirksamkeitsüberzeugungen Grundschullehramtsstudierender im Bereich der politischen Bildung
}

\author{
Barbara Lenzgeiger
}

Eingegangen: 28. Februar 2021 / Angenommen: 26. August 2021 / Online publiziert: 11. Januar 2022 (C) Der/die Autor(en) 2022

Zusammenfassung Es gibt nur wenige Erkenntnisse zu den Ausprägungen von Lehrkraftinteresse und Lehrkraftselbstwirksamkeitsüberzeugungen (angehender) Grundschullehrkräfte im Bereich der politischen Bildung. Offen bleibt außerdem, inwieweit sich Interesse und Selbstwirksamkeitsüberzeugungen von angehenden Grundschullehrkräften verändern lassen. Zur Beantwortung dieser Frage wurden im Rahmen einer quasi-experimentellen Interventionsstudie Daten von $N=145$ Studierenden des Lehramts für die Grundschule erhoben. Die Ergebnisse aus Strukturgleichungsmodellen und Regressionsanalysen zeigen, dass sich die Lehrkraftselbstwirksamkeitsüberzeugungen im Bereich der politischen Bildung durch eine Interventionsmaßnahme in Form eines Seminars verändern lassen. Jedoch waren keine Veränderungen des Lehrkraftinteresses der angehenden Lehrkräfte im Rahmen der Studie möglich. Gründe für diese Ergebnisse sowie Konsequenzen für die Ausbildung von Grundschullehrkräften werden diskutiert.

Schlüsselwörter Lehrkraftinteresse - Lehrkraftselbstwirksamkeitsüberzeugungen · Politikbezogener Sachunterricht · Lehrkraftausbildung

Dr. Barbara Lenzgeiger $(\triangle)$

Lehrstuhl für Grundschulpädagogik und Grundschuldidaktik, Department für Pädagogik und Rehabilitation, Fakultät für Psychologie und Pädagogik, Ludwig-Maximilians-Universität München, Leopoldstraße 13, 80802 München, Deutschland

E-Mail: barbara.lenzgeiger@1mu.de 


\title{
Changeability of teacher interest and teacher self-efficacy among primary school teachers-in-training in the field of political education
}

\begin{abstract}
Previous research focused on the specificity of teacher interest and teacher self-efficacy within the field of political education, primarily at the secondary school level. An open scientific question remains regarding the changeability of these constructs among primary school teachers-in-training. This quasi-experimental intervention study evaluated teacher interest and teacher self-efficacy among $N=145$ teachers-in-training within the field of political education at the primary school level. The results of structure equation modelling and latent regression analysis indicate that teachers' self-efficacy can be changed through a teaching intervention such as a university course; however, teacher interest could not be modified. The findings, limitations of the study and consequences for teacher training are discussed.
\end{abstract}

Keywords Teacher interest · Teacher self-efficacy · Political education · Teacher education

\section{Einleitung}

Um Schülerinnen und Schüler für politische Aufgaben- und Problemfelder zu sensibilisieren, ihr Interesse an politisch-gesellschaftlichen Fragestellungen zu wecken und ihnen zu ermöglichen, am demokratischen Leben teilzunehmen, kommt der Lehrkraft die Aufgabe zu, politisches Lernen im Sachunterricht so zu planen, zu gestalten und zu reflektieren, dass die Schülerinnen und Schüler bestmöglich in ihrem Kompetenzerwerb unterstützt werden. In der jüngeren Vergangenheit wurde postuliert, dass Lehrkräfte ohne ein vollständiges politikwissenschaftliches Studium diesen Anforderungen nicht umfassend gerecht werden (z. B. Massing 2007). Diese Einschätzung erfährt im Hinblick auf die politische Bildung im Sachunterricht in der Grundschule besondere Brisanz, da ein Großteil der bayerischen Grundschullehrkräfte kein fachspezifisches Studium der Politik aufweist. So bleibt der curriculare Rahmen des Sachunterrichts an bayerischen Universitäten für viele Grundschullehramtsstudierende oftmals der einzige Berührungspunkt mit politischer Bildung, wobei es im Ermessen der jeweiligen Dozierenden liegt, wie viel Raum die politische Bildung in den einzelnen Lehrveranstaltungen einnimmt. Obwohl verschiedene Studien auf die Bedeutung der universitären Ausbildung für die Professionalitätsentwicklung im Sachunterricht verweisen (z. B. Hartmann 2018), fehlt es an empirischen Erkenntnissen, inwieweit Aspekte der Professionalität wie Lehrkraftinteresse und Lehrkraftselbstwirksamkeitsüberzeugungen im Bereich der politischen Bildung verändert werden können. 


\section{Stand der Forschung}

\subsection{Die Bedeutung von Lehrkraftinteresse und Lehrkraftselbstwirksamkeitsüberzeugungen}

Wie eine Lehrkraft mit Herausforderungen im Schulalltag umgeht, hängt in besonderer Weise von ihrer Motivation ab, welche - neben Professionswissen, Überzeugungen und Werthaltungen sowie selbstregulativen Fähigkeiten - einen zentralen Teil professioneller Lehrkraftkompetenz ausmacht (Baumert und Kunter 2006). In der Erwartungs-Wert-Theorie (Atkinson 1957) wird davon ausgegangen, dass Personen in ihre Handlungsentscheidungen Überlegungen zur Erfolgswahrscheinlichkeit (Erwartung) sowie zur Attraktivität des jeweiligen Ziels (Wert) einbeziehen. In der Lehrkraftmotivationsforschung werden daher unter dem Begriff der motivationalen Orientierungen verschiedene Konstrukte verortet, die sowohl die Wertals auch die Erwartungskomponente berücksichtigen (Artelt und Kunter 2019). So werden die Konstrukte Lehrkraftinteresse und Lehrkraftselbstwirksamkeitsüberzeugungen als zentrale Aspekte motivationaler Orientierungen betrachtet, welche das unterrichtliche Handeln der Lehrkräfte beeinflussen (Kunter 2011).

Im deutschsprachigen Raum liegt den meisten Arbeiten, die sich mit Lehrkraftinteresse beschäftigen, die Konzeption der Person-Gegenstands-Theorie der Münchner Gruppe um Hans Schiefele (1974) mit den Erweiterungen der Forschergruppe um Andreas Krapp (2005) zugrunde. So wird unter Interesse die spezielle und relativ stabile Beziehung einer Person zu einem Gegenstand verstanden, welche sich durch positive Emotionen, einen verbesserten Bezugswert und erkenntnistheoretische Orientierung auszeichnet. Ausgehend hiervon kann Lehrkraftinteresse fachspezifisch modelliert und in Unterrichtsinteresse (berufliches Interesse) und Fach-/ Sachinteresse $^{1}$ (privates, außerschulisches Interesse) gegliedert werden (Lange 2010; Büchel 2019).

Während Lehrkraftinteresse vor allem die Wertkomponente von Motivation beschreibt, schließen Selbstwirksamkeitsüberzeugungen an die Erwartungskomponente an. Zur Beschreibung von Lehrkraftselbstwirksamkeitsüberzeugungen orientieren sich aktuelle Arbeiten vorranging an der sozial-kognitiven Theorie Banduras (1997) und dessen Modellierung der Allgemeinen Selbstwirksamkeitsüberzeugungen. Somit werden unter Lehrkraftselbstwirksamkeitsüberzeugungen die Überzeugungen einer Lehrkraft darüber verstanden, wie gut es ihr selbst gelingen kann, das Lernen und Verhalten ihrer Schülerinnen und Schüler zu unterstützen - auch wenn Schülerinnen und Schüler, Inhalte oder andere Aspekte des Unterrichtens sie selbst vor eine Herausforderung stellen (Tschannen-Moran und Woolfolk Hoy 2001; Schwarzer und Jerusalem 2002). Dabei können sich Lehrkraftselbstwirksamkeitsüberzeugungen auch auf einen Fachbereich beziehen (Warner und Schwarzer 2009).

Im Rahmen der Lehrkraftinteressensforschung zeigte sich, dass sowohl das Fachinteresse als auch das Unterrichtsinteresse der Lehrkräfte positive Auswirkungen auf die Gestaltung des Unterrichts haben. So ergaben sich nicht nur positive Zusammenhänge mit dem geplanten Einsatz und der Bereitschaft der Lehrkräfte, die

\footnotetext{
1 In Anlehnung an Baumert und Kunter (2006) wird im Folgenden der Begriff Fachinteresse verwendet.
} 
eigenen Interessen weiterzuentwickeln, sondern auch mit dem tatsächlichen Unterrichtshandeln, wie mit der Art der Strukturierung des Unterrichts oder dem Einsatz forschungsbasierter Methoden (z. B. Franz 2008; Eren 2012). Weniger eindeutig ist die Forschungslage auf Ebene der Schülerinnen und Schüler. Während Long und Woolfolk Hoy (2006) von positiven Auswirkungen auf die Motivation und Leistung der Schülerinnen und Schüler berichten, konstatiert Lange (2010) als Fazit ihrer Untersuchungen im naturwissenschaftlichen Bereich in der Grundschule, dass der Effekt des Fachinteresses der Lehrkraft auf die Leistungen der Schülerinnen und Schüler nicht signifikant sei. Dennoch gibt es Hinweise auf Zusammenhänge zwischen Unterrichtsinteresse und Selbstwirksamkeitsüberzeugungen von Lehrkräften (Franz 2008; Sorge et al. 2019).

Ähnliche Ergebnisse auf Lehrkraftebene finden sich auch in Untersuchungen zu den Lehrkraftselbstwirksamkeitsüberzeugungen. So wirken sich hohe berufliche Selbstwirksamkeitsüberzeugungen von Lehrkräften positiv auf die Wahrnehmung der eigenen Situation, wie die Berufszufriedenheit und Einstellungen (Caprara et al. 2006), sowie die Bereitschaft für berufliches und außerunterrichtliches Engagement aus (z.B. Schmitz und Schwarzer 2002). Lehrkräfte mit hohen Selbstwirksamkeitsüberzeugungen setzen ihren Schülerinnen und Schülern höhere Lernziele, verwenden innovativere und effektivere Methoden, unterstützen ihre Schülerinnen und Schüler wirkungsvoller und haben eine bessere Beziehung zu diesen (z.B. Morris-Rothschild und Brassard 2006; Lakshmanan et al. 2011). Auf Ebene der Schülerinnen und Schüler zeigen sich Zusammenhänge zwischen Lehrkraftselbstwirksamkeitsüberzeugungen mit Einstellungen, Motivation und Leistungen der Schülerinnen und Schüler (z. B. Caprara et al. 2006).

\subsection{Entwicklung von Lehrkraftinteresse und Lehrkraftselbstwirksamkeitsüberzeugungen}

Wenn es um die Frage der Veränderung von Lehrkraftinteresse und Lehrkraftselbstwirksamkeitsüberzeugungen gehen soll, muss zunächst deren Entwicklung in den Blick genommen werden.

Nach Hidi und Renninger (2006) handelt es sich bei der Entwicklung des individuellen Interesses um einen langwierigen Prozess, der verschiedene Phasen durchläuft und je nach Phase unterschiedlich stark durch Anstrengung, Selbstwirksamkeit, Zielorientierung und Selbstregulierung beeinflusst wird. Dabei nehmen Hidi und Renninger an, dass Interesse relativ stabil ist. Die Forschungslage hierzu ist allerdings uneinheitlich. Während in manchen Studien berichtet wird, dass Veränderungen beim Fachinteresse und beim Unterrichtsinteresse möglich sind (z. B. HeinrichDönges 2016), kamen andere Untersuchungen zum Schluss, dass das Fachinteresse stabil ist und im Rahmen dieser Studien nicht verändert werden konnte (z.B. Kleickmann et al. 2006).

Klarer scheint die Forschungslage zu den Lehrkraftselbstwirksamkeitsüberzeugungen. Ausgehend von Bandura (1997) beschrieben Tschannen-Moran und Woolfolk Hoy (2001) vier wesentliche Einflussfaktoren, die die Bewertung der eigenen Kompetenzen beeinflussen: eigene Handlungserfahrungen, physiologische und affektive Reaktionen, Handlungsbeobachtungen sowie verbale und soziale Beein- 
flussungen. Der Einfluss dieser Faktoren auf die Selbstwirksamkeitsüberzeugungen variiert dabei je nach bestehenden Überzeugungen und der Bewertung der neuen Informationen. Im Rahmen verschiedener Studien konnte gezeigt werden, dass Selbstwirksamkeitsüberzeugungen (angehender) Lehrkräfte durch Seminare oder Workshops gezielt verändert werden können, wobei der Fokus der Interventionen vor allem auf Elementen forschend-entdeckenden Lernens, selbstreflexiven Phasen sowie unterrichtsmethodischen Aspekten lag (z. B. Lakshmanan et al. 2011; Hartell et al. 2015).

\subsection{Lehrkraftinteresse und Lehrkraftselbstwirksamkeitsüberzeugungen im Bereich der politischen Bildung}

Im Bereich der politischen Bildung liegen bisher nur wenige Befunde zu den Ausprägungen motivationaler Orientierungen vor.

Die Forschergruppe um Oberle untersuchte im Rahmen der PKP-Studie mit einem Paper-Pencil-Test motivationale Orientierungen von Lehramtsstudierenden, Referendarinnen und Referendaren und Lehrkräften mit dem Unterrichtsfach Politik in den Schularten Grundschule, Hauptschule, Realschule und im Gymnasium. Dabei wurden die Grundschul-, Hauptschul- und Realschullehrkräfte (GHR-Lehrkräfte) zu einer Kohorte zusammengefasst, weswegen keine grundschulspezifischen Aussagen getroffen werden können. Das politische Fachinteresse der (angehenden) GHR-Lehrkräfte ist eher gering (Oberle et al. 2013). Diese Befunde konnten im Rahmen einer schriftlichen Befragung im Primarbereich repliziert werden (Näther und Merkens 2012). Dabei ist jedoch zu berücksichtigen, dass in der primarspezifischen Studie nicht alle Teilkomponenten des Interessenskonstrukts erhoben wurden. Hinsichtlich der Auswirkungen des Interesses auf Lehrkraftebene wurden Zusammenhänge des politischen Fachinteresses der befragten Gymnasiallehrkräfte mit ihrem politischen Fachwissen festgestellt. Bei den befragten GHR-Lehrkräften zeigten sich solche Zusammenhänge nicht (Weschenfelder 2014).

Es gibt Befunde, dass Politikstudierende positive Selbstwirksamkeitsüberzeugungen hinsichtlich ihrer künftigen Tätigkeiten als Politiklehrkräfte aufweisen (Oberle et al. 2013; Kalcsics et al. 2015). Zudem wurde festgestellt, dass geringe Selbstwirksamkeitsüberzeugungen hinsichtlich der Gestaltung des Unterrichts mit einem erhöhten Belastungserleben der Politiklehrkräfte zusammenhängen (Oberle et al. 2014).

\section{Forschungsdesiderate, Fragestellungen und Hypothesen}

Der Stand der Forschung zeigt, dass Lehrkraftinteresse und Lehrkraftselbstwirksamkeitsüberzeugungen aufgrund der implizit angenommenen Wirkungskette von den motivationalen Orientierungen der Lehrkräfte über die Gestaltung des Unterrichts auf Schülerinnen- und Schülermotivation und -leistungen eine besondere Bedeutung zukommen. Mit Blick auf die ausgeführten Forschungsbefunde ergeben sich für die durchgeführte Untersuchung folgende Forschungsfragen und Hypothesen: 
1. Können das Fachinteresse sowie das Unterrichtsinteresse an politischen Inhalten von Grundschullehramtsstudierenden durch eine Interventionsmaßnahme verändert werden?

$H_{1}$ : Das Unterrichtsinteresse kann durch die Interventionsmaßnahme verändert werden, das Fachinteresse bleibt stabil.

2. Können politische Lehrkraftselbstwirksamkeitsüberzeugungen von Grundschullehramtsstudierenden durch eine Interventionsmaßnahme verändert werden?

$\mathrm{H}_{2}$ : Die Lehrkraftselbstwirksamkeitsüberzeugungen können durch die Interventionsmaßnahme verändert werden.

3. Durch welche (weiteren) untersuchten Merkmale (politisches Fachinteresse und Unterrichtsinteresse, politische Lehrkraftselbstwirksamkeitsüberzeugungen, $\mathrm{Zu}$ gehörigkeit zur Experimentalgruppe) zum Prä-Messzeitpunkt lässt sich eine Veränderung beim Fachinteresse sowie beim Unterrichtsinteresse politischer Inhalte erklären?

$H_{3}$ : Das Unterrichtsinteresse an politischen Inhalten zum Post-Messzeitpunkt lässt sich durch politische Lehrkraftselbstwirksamkeitsüberzeugungen (Prä), politisches Fachinteresse (Prä) und Unterrichtsinteresse (Prä) sowie die Zugehörigkeit zur Experimentalgruppe erklären. Das Fachinteresse an politischen Inhalten zum Post-Messzeitpunkt lässt sich durch politische Lehrkraftselbstwirksamkeitsüberzeugungen (Prä) sowie politisches Fachinteresse (Prä) und Unterrichtsinteresse (Prä) erklären.

4. Durch welche (weiteren) untersuchten Merkmale (politisches Fachinteresse und Unterrichtsinteresse, politische Lehrkraftselbstwirksamkeitsüberzeugungen, $\mathrm{Zu}$ gehörigkeit zur Experimentalgruppe) zum Prä-Messzeitpunkt lässt sich eine Veränderung bei den politischen Lehrkraftselbstwirksamkeitsüberzeugungen erklären?

$H_{4}$ : Politische Lehrkraftselbstwirksamkeitsüberzeugungen zum Post-Messzeitpunkt lassen sich durch politische Lehrkraftselbstwirksamkeitsüberzeugungen (Prä), politisches Fachinteresse (Prä) und Unterrichtsinteresse (Prä) sowie die Zugehörigkeit zur Experimentalgruppe erklären.

\section{Methodisches Vorgehen}

\subsection{Untersuchungsdesign}

Die Daten zur Beantwortung der Forschungsfragen stammen aus einer quasi-experimentellen Interventionsstudie im Prä-Post-Design, in dem zwei Gruppen - Experimentalgruppe (EG) und Kontrollgruppe (KG) - zu zwei Messzeitpunkten (Prä/ vor der Intervention: zu Beginn des Semesters, Post/ nach der Intervention: am Ende des Semesters) mittels Fragebogen befragt wurden.

Die Intervention bestand aus einem Sachunterrichtsseminar, welches 12 bzw. 13 Sitzungen umfasste. Die Gestaltung der drei Seminare, welche die Studierenden der Experimentalgruppe als Interventionsmaßnahme besuchten, gründete auf Erkenntnissen zum reflexiven (z. B. Lerntagebücher) und forschenden Lernen (z. B. Befragung von Schülerinnen und Schüler), da sich bereits in anderen Domänen ge- 
zeigt hatte, dass Veränderungen motivationaler Orientierungen möglich sind (siehe Abschn. 2.2). Gegenstand der Seminare waren politische Kompetenzmodelle, typische politische Präkonzepte sowie theoretische Grundlagen und konkrete Ansätze, politische Themen im Sachunterricht umzusetzen (GDSU 2013). ${ }^{2}$ Die Studierenden der Kontrollgruppe nahmen an einem von drei Sachunterrichtsseminaren teil, dessen Schwerpunkt nicht auf politischen Inhalten lag.

\subsection{Erhebungsinstrumente}

Die motivationalen Orientierungen wurden mit Hilfe einer vierstufigen Likert-Skala erfasst. Dabei konnten Instrumente anderer Studien teilweise in modifizierter Form genutzt werden. So wurden die Lehrkraftselbstwirksamkeitsüberzeugungen in Anlehnung an die Skala zur Lehrkraftselbstwirksamkeit von Schwarzer und Schmitz (1999) formuliert. Die Ursprungsitems, welche keinen Fachbezug aufweisen, wurden dabei um das politische Lernen im Rahmen der sozialwissenschaftlichen Perspektive des Sachunterrichts ergänzt (Beispielitem: Ich bin mir sicher, dass ich kreative Ideen entwickeln kann, um politische Themen umzusetzen). Dabei gilt die Annahme, dass der Fachbereich den eigentlichen Handlungsrahmen der (angehenden) Lehrkräfte bildet und deswegen fachbereichsspezifische Selbstwirksamkeitsüberzeugungen die Untersuchungsfrage besser und genauer erklären (Oser und Baeriswyl 2002). Es wurden nicht alle Items der Skala zur Lehrkraftselbstwirksamkeit von Schwarzer und Schmitz (1999) übernommen, sondern diese aus forschungsökonomischen Gründen in Anlehnung an den COACTIV-Fragenbogen (Baumert et al. 2009) auf drei Items gekürzt.

Das individuelle Lehrkraftinteresse der Studierenden wurde anhand der Subskalen Unterrichtsinteresse (Beispielitem: Ich finde es spannend, etwas über politikdidaktische Methoden (z. B. Dilemmata, Rollenspiel) zu erfahren) und Fachinteresse (Beispielitem: Ich lese gerne Artikel über politische Themen) untersucht. Dabei bezieht sich das Unterrichtsinteresse (fünf Items) speziell auf politische Bildung im Sachunterricht. Fachinteresse (sechs Items) hingegen umfasst das Interesse an politischen Sachthemen außerhalb des schulischen Kontextes. Die Unterscheidung zwischen Fachinteresse und Unterrichtsinteresse wird häufig im naturwissenschaftlichen Kontext angewandt (Franz 2008; Lange 2010). Um das Konstrukt Lehrkraftinteresse in allen Facetten abzubilden, wurden in die Items verschiedene Bestimmungsmerkmale von Interesse integriert (siehe Abschn. 2.2). Grundlegende Orientierung bei der Itemformulierung boten die Skalen zum Lehrkraftinteresse von Franz (2008) und Kleickmann et al. (2006).

Neben den motivationalen Orientierungen wurden auch Hintergrundvariablen wie das Geburtsjahr, die Semesterzahl und die Belegung des Fachs Sozialkunde erfasst. Da die Anzahl männlicher Teilnehmer sehr gering war, wurde das Geschlecht nicht ausgewertet, um Anonymität zu garantieren.

\footnotetext{
2 Weitere Informationen zur Intervention finden sich in Reichhart (2018).
} 
Tab. 1 Stichprobenkennwerte der Experimentalgruppe und der Kontrollgruppe

\begin{tabular}{lll}
\hline & $\begin{array}{l}\text { Experimentalgruppe } \\
(\mathrm{EG})\end{array}$ & $\begin{array}{l}\text { Kontrollgruppe } \\
(\mathrm{KG})\end{array}$ \\
\hline$n$ & 76 & 69 \\
Alter: $M(S D)$ & $22,87(1,99)$ & $23,05(1,92)$ \\
Semester: $M(S D)$ & $4,87(1,42)$ & $5,07(1,20)$ \\
Sozialkunde als Fach & $13 \%$ & $10 \%$ \\
Lehrkraftinteresse Prä $^{\mathrm{a}}(S D)$ & $0,49(0,22)$ & $0,37(0,21)$ \\
Lehrkraftselbstwirksamkeit Prä $^{\mathrm{a}}(S D)$ & $0,59(0,18)$ & $0,57(0,18)$ \\
Lehrkraftinteresse Post $^{\mathrm{a}}(S D)$ & $0,50(0,22)$ & $0,36(0,21)$ \\
Lehrkraftselbstwirksamkeit Post $^{\mathrm{a}}(S D)$ & $0,69(0,14)$ & $0,56(0,19)$ \\
\hline
\end{tabular}

$n$ Anzahl der Studierenden, $M$ Mittelwert, SD Standardabweichung, Prä Prä-Messzeitpunkt, Post PostMesszeitpunkt

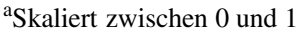

\subsection{Stichprobe}

Grundlage für die Ergebnisse sind Daten von $N=145$ (Experimentalgruppe: $n=76$, Kontrollgruppe: $n=69$ ) Studierenden, die zu beiden Messzeitpunkten an den Erhebungen teilnahmen. Die Stichprobe bestand aus Studierenden des Lehramts für Grundschulen einer bayerischen Universität. Die Teilnehmenden beider Gruppen waren zum ersten Erhebungszeitpunkt durchschnittlich 23 Jahre alt und studierten im 5. Semester (siehe Tab. 1). Um zu überprüfen, ob zwischen Kontrollgruppe und Experimentalgruppe signifikante demographische Unterschiede bestehen, wurden einfaktorielle Varianzanalysen gerechnet. Vor der Intervention zeigten sich keine signifikanten Unterschiede zwischen den beiden Gruppen hinsichtlich des Alters, der Semesterzahl oder der Anzahl der Studierenden, die Sozialkunde belegten. Während auch bei den politischen Lehrkraftselbstwirksamkeitsüberzeugungen zum Zeitpunkt des Prätests keine signifikanten Unterschiede vorlagen, ließen sich diese allerdings beim Lehrkraftinteresse (Unterrichtsinteresse, Fachinteresse) an Politik feststellen. Mit einer Differenz der Mittelwerte von 0,11 $(p<0,01)$ und einer berechneten Effektstärke von $d=0,27$ stellt dies einen kleinen Effekt dar (Cohen 1977). Eine mögliche Ursache hierfür ist die freie Wahl des Seminars. Da die Studierenden der Experimentalgruppe das Seminar zum politischen Lernen im Sachunterricht aus einem Angebot freiwillig ausgewählt hatten, liegt nahe, dass diese Studierenden ein gröBeres Interesse an Politik und dem Unterrichten politischer Inhalte zeigten.

\subsection{Analyseverfahren}

In einem ersten Schritt wurden klassische testtheoretische Item-Analysen durchgeführt. Die zunächst forcierte Gliederung in die Skalen Unterrichtsinteresse und Fachinteresse konnte durch eine explorative Faktorenanalyse nicht bestätigt werden. Deswegen wird im Folgenden ausschließlich von politischem Lehrkraftinteresse als einem Gesamtkonstrukt gesprochen.

Sowohl bei der Skala zu den politischen Lehrkraftselbstwirksamkeitsüberzeugungen $\left(r_{\text {it(min-max }}=0,47-0,55, \alpha=0,70\right)$ als auch bei der Skala zum Lehrkraftinteres- 
se $\left(r_{\mathrm{it}(\min -\max )}=0,60-0,87, \alpha=0,95\right)$ im Bereich politischer Bildung kann die interne Konsistenz als zufriedenstellend, für die Skala Lehrkraftinteresse mit $\alpha>0,90$ sogar als hoch bewertet werden.

Um Aussagen über das Verhältnis der Varianz zwischen den Seminaren treffen zu können, wurde der Intraklassenkoeffizient (ICC) berechnet. Während zum ersten Messzeitpunkt keine bedeutsamen Intraklassen-Korrelationen festgestellt werden können, wird beim zweiten Messzeitpunkt mit ICCs $>0,10$ bei beiden Skalen deutlich, dass die Zugehörigkeit zu einem der sechs Seminare - unabhängig davon, ob es sich um Seminare der Kontroll- oder Experimentalgruppe handelt - Einfluss auf die abhängigen Variablen der jeweiligen Gruppe hat. Daher wurde die geclusterte Struktur der Daten berücksichtigt, indem die Zugehörigkeit zu den einzelnen Seminaren als Prädiktor aufgenommen wurde.

Da der größte Anteil fehlender Werte auf Studierendenebene über beide Messzeitpunkte bei einem Item mit maximal drei fehlenden Werten $(1,03 \%)$ sehr klein ist und sich die Studierenden mit fehlenden Werten nicht von den Probanden unterscheiden, bei denen keine fehlenden Werte vorliegen (Little's MCAR-Test: $\chi^{2}=0,00$; $d f=1068 ; p=1,00)$, wurde multiple Imputation für unnötig erachtet und die fehlenden Werte mittels EM-Schätzer imputiert (Lüdtke et al. 2007).

Zur Beantwortung der Forschungsfragen wurden in einem ersten Schritt Strukturgleichungsmodelle herangezogen, da diese in Prä-Post-Designs Messfehler bei Messwiederholungen, Korrelationen und den unabhängigen Variablen berücksichtigen und im Gegensatz zu Varianzanalysen keine Vorannahmen hinsichtlich des Zusammenhangs treffen sowie treatment-Effekte besser nachweisen können (Mun et al. 2009). Dabei wurde zunächst der ML-Schätzer verwendet, der die Daten verhältnisskaliert betrachtet. Aufgrund besserer Fit-Indizes (siehe Kap. 5) wurde in den weiteren Berechnungen als Schätzmethode stattdessen der WLSMV-Schätzer herangezogen, der die Daten ordinal behandelt. Dies entspricht zudem der Argumentation der Autorengruppe um Cohen et al. (2007), die dazu rät, Daten aus Likert-skalierten Fragebogen stets mit nicht-parametrischen Tests zu analysieren.

Aufgrund der nicht durchgehend zufriedenstellenden Fit-Indizes der Strukturgleichungsmodelle wurden in einem weiteren Schritt zudem Regressionsanalysen durchgeführt. Als Prädiktoren für die politischen Lehrkraftselbstwirksamkeitsüberzeugungen zum zweiten Messzeitpunkt wurden in Modell 1 die Lehrkraftselbstwirksamkeitsüberzeugungen zum ersten Messzeitpunkt (Prä), das Lehrkraftinteresse zum ersten Messzeitpunkt (Prä) und die Zugehörigkeit zum Seminar aufgenommen. In Modell 2 wurde zusätzlich die Zugehörigkeit zur Experimentalgruppe aufgenommen. Zur Vorhersage des Lehrkraftinteresses wurden die entsprechenden Modelle analog konzipiert.

Die deskriptiven Skalenanalysen, die explorative Faktorenanalyse und die Schätzung der fehlenden Werte wurden mit SPSS Version 26 (IBM 2019) durchgeführt, die Berechnungen der ICCs mit MPlus Version 5.21 (2002) und die Strukturgleichungsmodelle und Regressionsanalysen mit RStudio Version 1.4.1103. Für die Strukturgleichungsmodelle wurde das Paket lavaan verwendet. 


\section{Ergebnisse}

Um die Einflüsse verschiedener Prädiktoren auf die motivationalen Orientierungen - Lehrkraftselbstwirksamkeitsüberzeugungen und Lehrkraftinteresse im Bereich der politischen Bildung - aufklären zu können, wurden die theoretischen Annahmen in ein Strukturgleichungsmodell überführt. Der $\chi^{2}$-Test wurde eingesetzt, um Aussagen über die Modellgüte des Strukturgleichungsmodells treffen zu können. Da der $\chi^{2}$-Test sensibel auf die Stichprobengröße reagiert (West et al. 2012) und im vorliegenden Strukturgleichungsmodell signifikant wird, wurden auch der CFI und der RSMEA sowie der SRMR eingehender untersucht. Während der RMSEA mit 0,04 und der CFI $<0,99$ eine gute Passung des Modells indizieren, kann der Wert des SRMR mit 0,08 noch als akzeptabel bezeichnet werden (Schermelleh-Engel und Moosbrugger 2003). Lediglich der signifikante $\chi^{2}$-Test ist nicht akzeptabel. Laut Maydeu-Olivares (2017) kann ein solcher Misfit als trivial behandelt werden, wenn der SRMR kleiner 0,10 und keine Kovarianz der Residuen größer als 0,20 ist. Zweiteres ist in diesem Modell nicht der Fall. Daher müssen Schlussfolgerungen aus diesem Modell trotz einer kleinen Effektstärke des Misfits vorsichtig und unter Vorbehalt interpretiert werden.

Das Pfadmodell (siehe Abb. 1) deutet an, dass sich die Teilnahme an der Interventionsmaßnahme auf die Lehrkraftselbstwirksamkeitsüberzeugungen $(\beta=0,38)$ und auf das Lehrkraftinteresse $(\beta=0,34)$ der Studierenden auswirkte. Während das Lehrkraftinteresse der Studierenden zum Zeitpunkt des Prätests einen positiven Einfluss auf die Lehrkraftselbstwirksamkeitsüberzeugungen zum Posttest hatte $(\beta=0,47)$, schienen die Lehrkraftselbstwirksamkeitsüberzeugungen zum Prätest keinen Einfluss auf das Lehrkraftinteresse zum Posttest zu haben $(\beta=-0,09)$.

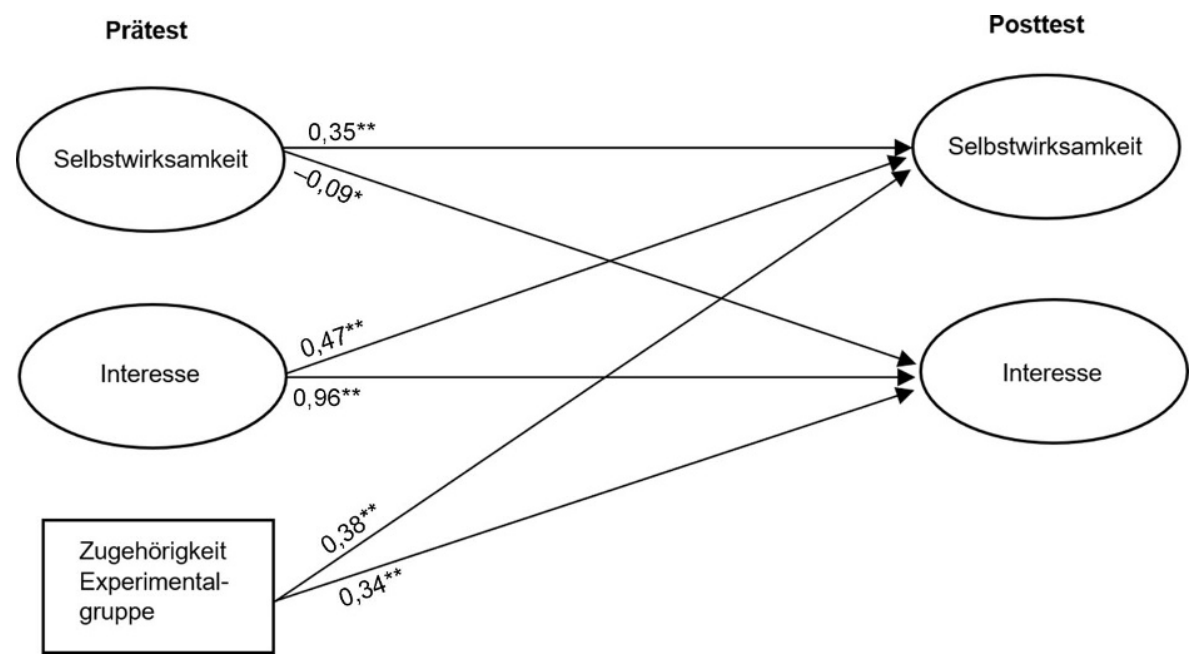

Abb. 1 Pfadmodell zur Abbildung der Einflussfaktoren auf die Lehrkraftselbstwirksamkeitsüberzeugungen und das Lehrkraftinteresse der Studierenden im Bereich der politischen Bildung. $N=145$. Berichtet werden die standardisierten Koeffizienten. ${ }^{* *} p<0,01,{ }^{*} p<0,05$, Modellfit: $\chi^{2}(370)=444,447, p=0,005$, CFI $>0,99$, SRMR $=0,08$, RMSEA $=0,04$ 
Tab. 2 Hierarchische multiple Regression zum politischen Lehrkraftinteresse (Post)

\begin{tabular}{lllllll}
\hline & Modell 1 & \multicolumn{3}{c}{ Modell 2 } \\
& $B$ & $S E$ & $\beta$ & $B$ & $S E$ & $\beta$ \\
\hline Intercept & 0,08 & 0,03 & - & 0,04 & 0,04 & - \\
Lehrkraftselbstwirksamkeit (Prä) & $-0,09$ & 0,05 & $-0,07$ & $-0,08$ & 0,06 & $-0,06$ \\
Lehrkraftinteresse (Prä) & 0,90 & 0,05 & $0,90^{* *}$ & 0,88 & 0,05 & $0,88^{* *}$ \\
EG & - & - & - & 0,08 & 0,03 & 0,37 \\
Multiple R & & & & 0,80 & & \\
Adjusted R & 0,79 & & & 0,79 & & \\
\hline
\end{tabular}

$N=145$

Prä Prä-Messzeitpunkt, Post Post-Messzeitpunkt, EG Zugehörigkeit zur Experimentalgruppe, $B$ nicht standardisierter Regressionskoeffizient, $S E$ Standardfehler, $\beta$ standardisierter Regressionskoeffizient $* * p<0,01, * p<0,05$

Tab. 3 Hierarchische multiple Regression zu den politischen Lehrkraftselbstwirksamkeitsüberzeugungen (Post)

\begin{tabular}{lllllll}
\hline & Modell 1 & \multicolumn{3}{c}{ Modell 2 } \\
& $B$ & $S E$ & $\beta$ & $B$ & $S E$ & $\beta$ \\
\hline Intercept & 0,34 & 0,04 & - & 0,27 & 0,05 & - \\
Lehrkraftselbstwirksamkeit (Prä) & 0,32 & 0,07 & $0,33^{* *}$ & 0,35 & 0,07 & $0,35^{* *}$ \\
Lehrkraftinteresse (Prä) & 0,29 & 0,06 & $0,36^{* *}$ & 0,26 & 0,06 & $0,32^{* *}$ \\
EG & - & - & - & 0,13 & 0,04 & $0,75^{* *}$ \\
Multiple R & & & & 0,49 & & \\
Adjusted R & 0,45 & & & 0,46 & & \\
\hline
\end{tabular}

$N=145$

Prä Prä-Messzeitpunkt, Post Post-Messzeitpunkt, EG Zugehörigkeit zur Experimentalgruppe, $B$ nicht standardisierter Regressionskoeffizient, $S E$ Standardfehler, $\beta$ standardisierter Regressionskoeffizient $* * p<0,01, * p<0,05$

Um eindeutigere Aussagen zu den Auswirkungen der Intervention treffen zu können, wurden Regressionsanalysen durchgeführt.

Die Regressionsanalysen (siehe Tab. 2) zeigen entgegen der Hypothese 1, dass beim Lehrkraftinteresse keine signifikanten Veränderungen festzustellen sind (Forschungsfrage 1). Durch Modell 2 können zwar insgesamt $80 \%$ der Varianz des Interesses zum Post-Testzeitpunkt erklärt werden, allerdings hat die Zugehörigkeit zur Experimentalgruppe und damit die Interventionsmaßnahme darauf keine Auswirkung. Einzig das Lehrkraftinteresse zum Prä-Testzeitpunkt hängt mit dem Lehrkraftinteresse zum Post-Testzeitpunkt zusammen $(\beta=0,88)$ (Forschungsfrage 3 ).

Signifikante Veränderungen hingegen waren bei den Lehrkraftselbstwirksamkeitsüberzeugungen festzustellen. Die Analysen (siehe Tab. 3) zeigen, dass sich - unter Berücksichtigung der Zugehörigkeit zum jeweiligen Seminar - durch die Lehrkraftselbstwirksamkeitsüberzeugungen zum Prä-Messzeitpunkt und durch das Lehrkraftinteresse zum Prä-Messzeitpunkt $45 \%$ der Varianz der Lehrkraftselbstwirksamkeitsüberzeugungen zum Post-Messzeitpunkt erklären lassen (Modell 1). Zudem wurde deutlich, dass die Studierenden der Experimentalgruppe zum Posttest signifikant höhere Lehrkraftselbstwirksamkeitsüberzeugungen im Bereich der politischen Bildung aufwiesen als beim Prätest $(\beta=0,75)$ (Forschungsfrage 2$)$. Dies bedeutet, dass sich 
zusätzlich 4\% der Varianz allein durch die Zugehörigkeit zur Experimentalgruppe und dadurch durch die Interventionsmaßnahme erklären lassen. Zur Varianzaufklärung trugen außerdem die Lehrkraftselbstwirksamkeitsüberzeugungen $(\beta=0,35)$ und das Lehrkraftinteresse $(\beta=0,32)$ zum Prä-Messzeitpunkt bei (Forschungsfrage 4$)$.

\section{Diskussion und Ausblick}

Die Ergebnisse der vorliegenden Studie geben erste Hinweise darauf, inwieweit es möglich ist, politische Lehrkraftselbstwirksamkeitsüberzeugungen und politisches Lehrkraftinteresse zu verändern.

Die Regressionsanalysen deuten an, dass es sich als schwierig gestaltet, Veränderungen des Lehrkraftinteresses im Bereich der politischen Bildung zu erwirken. Dies deckt sich mit den Befunden anderer Domänen, in welchen es ebenfalls nicht gelang, Lehrkraftinteressen zu verändern (Kleickmann et al. 2006; Martschinke 2008). Dabei liegt die Ursache möglicherweise in der Struktur des Konstrukts Lehrkraftinteresse. So scheint - wie bereits Eren (2012) vermutete - Lehrkraftinteresse dem individuellen Interesse sehr nahe zu sein und daher relativ stabile Strukturen aufzuweisen. Denkbar wäre, dass ein einziges Seminar nicht ausreicht, um Interessen nachhaltig zu verändern und längerfristige Maßnahmen gefragt sind oder in die Konzeption des Seminars noch stärker Maßnahmen integriert werden sollten, die das Potenzial haben, Lehrkraftinteresse zu verändern.

Bei den politischen Lehrkraftselbstwirksamkeitsüberzeugungen hingegen waren - wie vermutet - Veränderungen möglich. Dabei scheint die Intervention auch längerfristig anzuhalten: Die befragten Studierenden wiesen auch sechs Monate nach der Intervention noch signifikant höhere politische Lehrkraftselbstwirksamkeitsüberzeugungen auf (Reichhart 2018). Dies deutet darauf hin, dass durch einen relativ geringen Aufwand, wie dem eines Seminars, Veränderungen bestimmter professioneller Kompetenzen möglich sein können und die universitäre Ausbildung eine zentrale Rolle in der Entwicklung professioneller Lehrkraftkompetenzen einnimmt. Dabei machen motivationale Orientierungen nur einen Aspekt der Lehrkraftprofessionalität aus. Vor dem Hintergrund der Bedeutung des fachdidaktischen Wissens für den Schulerfolg von Schülerinnen und Schülern (Lange et al. 2012) sollten daher in weiteren Studien auch andere professionelle Lehrkraftkompetenzen im Bereich der politischen Bildung in den Blick genommen werden.

Interessant erscheint in diesem Zusammenhang auch das Zusammenspiel von politischem Lehrkraftinteresse und Lehrkraftselbstwirksamkeitsüberzeugungen. Während sich das Lehrkraftinteresse der befragten Studierenden beim Prä-Messzeitpunkt auf die Lehrkraftselbstwirksamkeitsüberzeugungen nach der Interventionsmaßnahme auswirkt, haben politische Lehrkraftselbstwirksamkeitsüberzeugungen zum PräMesszeitpunkt keine Auswirkungen auf das Lehrkraftinteresse. Somit scheint dem politischen Lehrkraftinteresse eine zentrale Bedeutung zuzukommen, die es im Rahmen weiterer Studien mit größeren Stichproben und einer veränderten Seminarstruktur genauer zu untersuchen gilt. Dabei wäre denkbar, den Schwerpunkt der Intervention stärker auf das forschende Lernen und damit auf eine Erhöhung schul- 
praktischer Anteile zu legen, da in anderen Domänen gezeigt werden konnte, dass dadurch Veränderungen möglich sind (Baumgardt 2014).

Bei der Interpretation der Ergebnisse muss berücksichtigt werden, dass es sich bei der Studie um eine Gelegenheitsstichprobe handelt, da die Studierenden nicht zufällig ausgewählt wurden. Einschränkend ist dabei zu beachten, dass das Selegieren von Stichproben, das sich in der Erhebung durch die Freiwilligkeit der Teilnahme ergab, zu einer Unterschätzung des Gesamtzusammenhangs führen kann. Damit einhergehend kann auch die relativ kleine Stichprobengröße von 145 Studierenden erklärt werden, die vor allem organisatorische Ursachen hat. Da die Anzahl der Gruppen in der vorliegenden Studie mit sechs Gruppen sehr gering ist, ist es möglich, dass die Standardfehler, die zur Berechnung der Varianz bei den Regressionsanalysen herangezogen werden, zu klein geschätzt wurden (Browne und Draper 2000). Um dies zu überprüfen, wurden daher weitere Regressionsanalysen gerechnet, in denen keine Clustervariablen berücksichtigt wurden. Hierbei wurde gezeigt, dass die Veränderung der Lehrkraftselbstwirksamkeitsüberzeugungen auch im Rahmen dieser Berechnungen signifikant ist. Ebenfalls organisatorischen Gründen ist die Tatsache geschuldet, dass die Interventionsmaßnahme durch die Versuchsleiterin durchgeführt wurde. Dies kann das Ergebnis eines psychologischen Versuchs beeinflussen, da es zu einer Verzerrung der Ergebnisse kommen kann (=Versuchsleitereffekt). Gleichwohl kann dadurch sichergestellt werden, dass diese Seminare gleich abliefen und somit die Durchführungsobjektivität gegeben war. Die anfängliche Annahme, dass Unterrichtsinteresse und Fachinteresse verschiedene Konstrukte sind, wurde durch die explorative Faktorenanalyse nicht bestätigt. Stattdessen lag hier nur eine latente Variable vor, die als Lehrkraftinteresse betitelt wurde. Insofern konnte im Rahmen der vorliegenden Studie nicht zwischen Unterrichtsinteresse und Fachinteresse differenziert werden.

Trotz dieser Limitationen kann ausgehend von den vorliegenden Befunden für eine intensivere Auseinandersetzung mit politischen Inhalten in der Lehrkraftausbildung plädiert werden. An den meisten bayerischen Universitäten scheint es aufgrund der begrenzten zeitlichen Vorgaben und der knappen (personellen) Ressourcen im Sachunterricht nicht möglich, Studierende so auszubilden, dass sie in der Lage sind, professionelle Kompetenzen in einzelnen Perspektiven des Sachunterrichts - insbesondere der politischen Bildung - zu entwickeln. Daher sind politische Steuerungsmaßnahmen gefragt, um Einfluss auf die Dauer und Organisation der Lehrkraftausbildung zu nehmen. So scheint es für den Sachunterricht sinnvoll, den einzelnen Perspektiven - und hierbei insbesondere der politischen Bildung - ein Mehr an Zeit zuzugestehen. Nur so kann allen (angehenden) Lehrkräften die Möglichkeit gegeben werden, Lehrkraftinteresse und Lehrkraftselbstwirksamkeitsüberzeugungen hinsichtlich der einzelnen Bereiche aufzubauen bzw. weiterzuentwickeln.

Funding Open Access funding enabled and organized by Projekt DEAL.

Open Access Dieser Artikel wird unter der Creative Commons Namensnennung 4.0 International Lizenz veröffentlicht, welche die Nutzung, Vervielfältigung, Bearbeitung, Verbreitung und Wiedergabe in jeglichem Medium und Format erlaubt, sofern Sie den/die ursprünglichen Autor(en) und die Quelle ordnungsgemäß nennen, einen Link zur Creative Commons Lizenz beifügen und angeben, ob Änderungen vorgenommen wurden. 
Die in diesem Artikel enthaltenen Bilder und sonstiges Drittmaterial unterliegen ebenfalls der genannten Creative Commons Lizenz, sofern sich aus der Abbildungslegende nichts anderes ergibt. Sofern das betreffende Material nicht unter der genannten Creative Commons Lizenz steht und die betreffende Handlung nicht nach gesetzlichen Vorschriften erlaubt ist, ist für die oben aufgeführten Weiterverwendungen des Materials die Einwilligung des jeweiligen Rechteinhabers einzuholen.

Weitere Details zur Lizenz entnehmen Sie bitte der Lizenzinformation auf http://creativecommons.org/ licenses/by/4.0/deed.de.

\section{Literatur}

Artelt, C., \& Kunter, M. (2019). Kompetenzen und berufliche Entwicklung von Lehrkräften. In D. Urhahne, M. Dresel \& F. Fischer (Hrsg.), Psychologie für den Lehrberuf (S. 395-418). Berlin: Springer.

Atkinson, J.W. (1957). Motivational determinants of risktaking behavior. Psychological Review, 64(6), 359-372.

Bandura, A. (1997). Self-efficacy. The exercise of control. New York: Freeman.

Baumert, J., \& Kunter, M. (2006). Stichwort: Professionelle Kompetenz von Lehrkräften. Zeitschrift für Erziehungswissenschaft, 9(4), 469-520.

Baumert, J., Blum, W., Brunner, M., Dubberke, T., Jordan, A., Klusmann, U., Krauss, S., Kunter, M., Löwen, K., Neubrand, M., \& Tsai, Y.-M. (2009). Materialien aus der Bildungsforschung. Nr. 83: Professionswissen von Lehrkräften, kognitiv aktivierender Mathematikunterricht und die Entwicklung von mathematischer Kompetenz (COACTIV). Dokumentation der Erhebungsinstrumente. Berlin: MaxPlanck-Inst. für Bildungsforschung.

Baumgardt, I. (2014). Forschen, Lehren und Lernen in der Lehrerausbildung. Fachdidaktische Beiträge aus der universitären Praxis. Baltmannsweiler: Schneider Verl. Hohengehren.

Browne, W. J., \& Draper, D. (2000). Implementation and performance issues in the Bayesian and likelihood fitting of multilevel models. Computational Statistics, 15, 391-420.

Büchel, S. (2019). Lehrermotivation im Sportunterricht. Effekte auf das Lernverhalten von Lehrpersonen und Unterrichtsprozesse. Wiesbaden: Springer.

Caprara, G. V., Barbaranelli, C., Steca, P., \& Malone, P. S. (2006). Teachers' self-efficacy beliefs as determinants of job satisfaction and students' academic achievment. A study at the school level. Journal of School Psychology, 44, 473-490.

Cohen, J. (1977). Statistical power analysis for the behavioral sciences. New York: Academic Press.

Cohen, L., Manion, L., \& Morrison, K. (2007). Research methods in education (6. Aufl.). London: Routledge.

Eren, A. (2012). Prospective teachers' interest in teaching, professional plans about teaching and career choice satisfaction: a relevant framework? Australian Journal of Education, 56(3), 303-318.

Franz, U. (2008). Forschung: Lehrer- und Unterrichtsvariablen im naturwissenschaftlichen Sachunterricht. Eine empirische Studie zum Wissenserwerb und zur Interessenentwicklung in der dritten Jahrgangsstufe. Bad Heilbrunn: Julius Klinkhardt.

GDSU (2013). Perspektivrahmen Sachunterricht. Bad Heilbrunn: Julius Klinkhardt.

Hartell, E., Gumaelius, L., \& Svärdh, J. (2015). Investigating technology teachers' self-efficacy on assessment. International Journal of Technology and Design Education, 25(3), 321-337.

Hartmann, C. (2018). Zur Bedeutsamkeit formaler Lerngelegenheiten für das fachdidaktische Wissen im Bereich der historischen Perspektive des Sachunterrichts. Zeitschrift für Grundschulforschung, 11(2), 269-284.

Heinrich-Dönges, A. (2016). Wie Lehrkräfte ihren Sachunterricht weiterentwickeln und Fortbildung sie dabei unterstützen kann. Ergebnisse einer empirischen Studie zur Identifikation von Gelingensbedingungen. In H. Giest, T. Goll \& A. Hartinger (Hrsg.), Sachunterricht - zwischen Kompetenzorientierung, Persönlichkeitsentwicklung, Lebenswelt und Fachbezug. Probleme und Perspektiven des Sachunterrichts, (Bd. 26, S. 116-123). Bad Heilbrunn: Julius Klinkhardt.

Hidi, S., \& Renninger, K. A. (2006). The four-phase model of interest development. Educational Psychologist, 41(2), 111-127.

Kalcsics, K., Schumann, S., Favre, P., \& Mathis, C. (2015). Die Rolle des fachdidaktischen Wissens von Lehrpersonen. Jahrestagung der Gesellschaft für Didaktik des Sachunterrichts. Dortmund.

Kleickmann, T., Möller, K., \& Jonen, A. (2006). Die Wirksamkeit von Fortbildungen und die Bedeutung von tutorieller Unterstützung. In R. Hinz \& T. Pütz (Hrsg.), Professionelles Handeln in der Grund- 
schule. Entwicklungslinien und Forschungsbefunde. Entwicklungslinien der Grundschulpädagogik, (Bd. 3, S. 121-128). Baltmannsweiler: Schneider Verlag Hohengehren.

Krapp, A. (2005). Psychologische Bedürfnisse und Interesse. Theoretische Überlegungen und parktische Schlussfolgerungen. In R. Vollmeyer \& J. C. Brunstein (Hrsg.), Motivationspsychologie und ihre Anwendung (1. Aufl. S. 23-38). Stuttgart: Kohlhammer.

Kunter, M. (2011). Motivation als Teil der professionellen Kompetenz. Forschungsbefunde zum Enthusiasmus von Lehrkräften. In M. Kunter, J. Baumert, W. Blum \& M. Neubrand (Hrsg.), Professionelle Kompetenz von Lehrkräften. Ergebnisse des Forschungsprogramms COACTIV (S. 259-275). Münster: Waxmann.

Lakshmanan, A., Heath, B. P., Perlmutter, A., \& Elder, M. (2011). The impact of science content and professional learning communities on science teaching efficacy and standards-based instruction. Journal of Research in Science Teaching, 48(5), 534-551.

Lange, K., Kleickmann, T., Tröbst, S., \& Möller, K. (2012). Fachdidaktisches Wissen von Lehrkräften und multiple Ziele im naturwissenschaftlichen Sachunterricht. Zeitschrift für Erziehungswissenschaft, 15, $55-75$.

Lange, K. (2010). Zusammenhänge zwischen naturwissenschaftsbezogenem fachspezifisch-pädagogischem Wissen von Grundschullehrkräften und Fortschritten im Verständnis naturwissenschaftlicher Konzepte bei Grundschülerinnen und -schülern. https://d-nb.info/1011948885/34. Zugegriffen: 4. Febr. 2021.

Long, J.F., \& Woolfolk Hoy, A. (2006). Interested instructors. A composite portrait of individual differences and effectiveness. Teaching and Teacher Education, 22, 303-314.

Lüdtke, O., Robitzsch, A., Trautwein, U., \& Köller, O. (2007). Umgang mit fehlenden Werten in der psychologischen Forschung. Psychologische Rundschau, 58(2), 103-117.

Martschinke, S. (2008). Selbstreflexives und forschendes Lernen in der Lehrerbildung. In A. Hartinger, R. Bauer \& R. Hitzler (Hrsg.), Veränderte Kindheit. Konsequenzen für die Lehrerbildung (S. 95-106). Bad Heilbrunn: Julius Klinkhardt.

Massing, P. (2007). Politische Bildung in der Grundschule. Überblick, Kritik, Perspektiven. In D. Richter (Hrsg.), Politische Bildung von Anfang an. Demokratie-Lernen in der Grundschule. Schriftenreihe/ Bundeszentrale für Politische Bildung, (Bd. 570, S. 18-35). Bonn: Bundeszentrale für Politische Bildung.

Maydeu-Olivares, A. (2017). Assessing the size of model misfit in structural equation models. Psychometrika, 82, 533-558.

Morris-Rothschild, B. K., \& Brassard, M.R. (2006). Teachers' conflict management styles. The role of attachment styles and classroom management efficacy. Journal of School Psychology, 44(2), 105-121.

Mun, E. Y., von Eye, A., \& White, H. R. (2009). An SEM approach for the evaluation of intervention effects using pre-post-post designs. Structural Equation Modeling, 16(2), 315-337.

Näther, T., \& Merkens, H. (2012). Auswertung der Befragung zu den Fortbildungspräferenzen von Grundschullehrenden. In H. Merkens (Hrsg.), Technikinteresse von Grundschullehrkräften (S. 38-74). Berlin.

Oberle, M., Weschenfelder, E., \& Weißeno, G. (2013). Motivationale Orientierungen angehender und praktizierender Politiklehrkräfte. In A. Besand (Hrsg.), Schriftenreihe der GPJE: Lehrer- und Schülerforschung in der politischen Bildung (S. 55-67). Schwalbach: Wochenschau.

Oberle, M., Weschenfelder, E., \& Weißeno, G. (2014). Beliefs als Element professioneller Kompetenz bei Politiklehrkräften in Deutschland. In B. Ziegler (Hrsg.), Vorstellungen, Konzepte und Kompetenzen von Lehrpersonen der politischen Bildung. Beiträge zur Tagung „Politische Bildung empirisch 2012“. Politische Bildung in der Schweiz, (Bd. 3, S. 124-137). Zürich: Rüegger.

Oser, F., \& Baeriswyl, F. J. (2002). Choreographies of teaching. Bridging instruction to learning. In V. Richardson (Hrsg.), Handbook of research on teaching (4. Aufl. S. 1031-1056). Washington, DC: American Educational Research Assoc.

Reichhart, B. (2018). Empirische Forschung in den gesellschaftswissenschaftlichen Fachdidaktiken: Lehrerprofessionalität im Bereich der politischen Bildung. Wiesbaden: Springer VS.

Schermelleh-Engel, K., \& Moosbrugger, H. (2003). Evaluating the fit of structural equation models. Tests of significance and descriptive goodness-of-fit measures. Methods of Psychological Research Online, $8(2), 23-74$.

Schiefele, H. (1974). Lernmotivation und Motivlernen. Grundzüge einer erziehungswissenschaftlichen Motivationslehre. München: Ehrenwirth.

Schmitz, G. S., \& Schwarzer, R. (2002). Individuelle und kollektive Selbstwirksamkeitserwartung von Lehrern. Zeitschrift für Pädagogik, 44(Beiheft), 192-214. 
Schwarzer, R., \& Jerusalem, M. (2002). Das Konzept der Selbstwirksamkeit. Zeitschrift für Pädagogik, 44(Beiheft), 28-53.

Schwarzer, R., \& Schmitz, G.S. (1999). Skala zur Lehrer-Selbstwirksamkeitserwartung (WIRKLEHR). In R. Schwarzer \& M. Jerusalem (Hrsg.), Skalen zur Erfassung von Lehrer- und Schülermerkmalen. Dokumentation der psychometrischen Verfahren im Rahmen der wissenschaftlichen Begleitung des Modellversuchs Selbstwirksame Schulen (S. 60-62). Berlin: ZPID.

Sorge, M., Keller, M., Neumann, K., \& Möller, J. (2019). Investigating the relationship between pre-service physics teachers' professional knowledge, self-concept, and interest. Journal of Research in Science Teaching, 56, 937-955.

Tschannen-Moran, M., \& Woolfolk Hoy, A. (2001). Teacher efficacy: capturing an elusive construct. Teaching and Teacher Education, 17, 783-805.

Warner, L., \& Schwarzer, R. (2009). Selbstwirksamkeit bei Lehrkräften. In O. Zlatkin-Troitschanskaia (Hrsg.), Lehrprofessionalität. Bedingungen, Genese, Wirkungen und ihre Messung. Beltz-Bibliothek. (S. 629-640). Weinheim: Beltz.

Weschenfelder, E. (2014). Professionelle Kompetenz von Politiklehrkräften. Eine Studie zu Wissen und Überzeugungen. Wiesbaden: Springer VS.

West, S. G., Taylor, A. B., \& Wu, W. (2012). Model fit and model selection in structural equation modeling. In R. H. Hoyle (Hrsg.), Handbook of structural equation modeling (S. 209-231). New York: Guilford. 